\title{
Incidência de lesões por pressão em Unidade de Terapia Intensiva em hospital com acreditação
}

\author{
Incidence of pressure injuries in Intensive Care Unit in an accredited hospital \\ Incidencia de las lesiones de presión en la Unidad de Cuidados Intensivos
de un hospital con acreditación
}

Anne Kayline Soares Teixeira', Tiago da Silva Nascimento², Ingrid Thaís Lopes de Sousa², Luis Rafael Leite Sampaio², Alessandra Rocha Mororó Pinheiro ${ }^{3}$

\begin{abstract}
RESUMO
As lesões por pressão são consideradas eventos adversos e uma complicação frequente em pacientes graves, refletindo de forma indireta a qualidade do cuidado. O presente estudo, de abordagem quantitativa, teve como objetivo analisar o perfil de incidência das lesões por pressão em Unidade de Terapia Intensiva de adultos, em 2014. A pesquisa, aprovada pelo Comitê de Ética (parecer 1.188.404), foi realizada em um hospital que possui 336 leitos, dos quais 21 são destinados à Unidade de Terapia Intensiva Adulto. Os dados foram coletados por meio de fichas de monitoramento de riscos referentes às incidências de lesões por pressão no período de janeiro a dezembro de 2014. Os dados foram transcritos, tabulados e organizados em tabelas de maio a junho de 2015 . Houve um total de 649 internações e foram identificadas 68 incidências, o que corresponde a 10,47\% dos casos de incidência sobre o total de internações. Destes, 51,5\% eram do sexo masculino e 48,5\% feminino; 58,8\% incidiram durante os primeiros 14 dias de internação. Predominou-se a faixa etária acima de 60 anos, com 79,4\%. Dos indivíduos com faixa etária acima de 60 anos, $61,1 \%$ apresentaram risco elevado para o desenvolvimento de lesão por pressão. A região sacral foi identificada em 46,4\%. 61,9\% dos casos eram de estágio 2. O estudo evidenciou baixa incidência de lesão por pressão nos pacientes da Unidade de Terapia Intensiva, levando-se em consideração o referencial de estudos internacionais e nacionais. Percebe-se, também, que predomina o seu surgimento em localizações anatômicas consideradas de maior pressão, e que as lesões foram identificadas precocemente.
\end{abstract}

DESCRITORES: Ferimentos e lesões. Úlcera por pressão. Unidades de Terapia Intensiva. Incidência. Estomaterapia.

\section{ABSTRACT}

Pressure injuries are considered adverse effects and a frequently observed complication in severely ill patients. They indirectly reflect the quality of nursing care. This is a quantitative study with the aim of analyzing the incidence profile of pressure injuries in an Intensive Care Unit for adults in the year of 2014. The study, approved by the Ethics Committee of the hospital (No. 1,188,404), was held in a hospital with 336 beds; 21 of these are intended for adult Intensive Care Unit. Data were collected using risk monitoring charts in order to calculate the incidence of pressure injuries from January to December of 2014. Data were transcribed, tabulated and organized in tables between May and June of 2015. There were 649 Intensive Care Unit admissions in the studied period and 68 patients with pressure injuries were identified, which corresponds to a 10,47\% incidence of cases over the total admissions. Among patients

1 Universidade Estadual do Ceará (UECE) - Fortaleza (CE), Brasil.

Endereço para correspondência: Departamento de Pós-Graduação em Cuidados Clínicos em Enfermagem e Saúde, Campus do Itaperi - Avenida Doutor Silas Munguba, 1.700 - CEP: 60714-242 - Fortaleza (CE), Brasil - E-mail: kaylinesoares@hotmail.com

¿Universidade de Fortaleza (UNIFOR) - Fortaleza (CE), Brasil.

${ }^{3}$ Escola de Saúde Pública do Ceará - Fortaleza (CE), Brasil.

Artigo recebido em: 16/11/2015 - Aceito para publicação em: 03/08/2016. 
with pressure injuries, $51.5 \%$ were male and $48.5 \%$ were female; $58.8 \%$ of the cases occurred during the first 14 days of Intensive Care Unit stay. 79.4\% of the patients were elderly, aged 60 years or older; in this group, $61.1 \%$ of them presented an increased risk for developing pressure injuries. The sacral region was the most frequent affected (46.4\%). Regarding categorization, stage 2 was the most prevalent (61.9\%). The present study evidenced an incidence of low-pressure injuries in intensive care patients, when compared to other studies, both national and international. It was also observed that pressure injuries most often happened in "high pressure" body regions, and that they presented an early identification.

DESCRIPTORS: Wounds and injuries. Pressure Ulcer. Intensive Care Unit. Incidence. Stomatherapy.

\section{RESUMEN}

Las lesiones de presión son consideradas eventos adversos y una complicación frecuente en pacientes en estado crítico. Las lesiones son un reflejo indirecto de la calidad del cuidado de las enfermeras. Se conduzco un estudio de abordaje cuantitativo, cuyo objetivo fue analizar el perfil de incidencia de las lesiones de presión en una Unidad de Cuidados Intensivos para adultos en 2014. La investigación, aprobada por el Comité de Ética (n 1.188.404), se realizó en un hospital con 336 camas; 21 en la Unidad de Cuidados Intensivos para adultos. Se recogieron los datos con fichas de seguimiento de riesgo que evaluaron las incidencias de lesiones de presión entre enero y diciembre 2014. Los datos fueron transcritos, tabulados y organizados en cuadros en los meses de mayo hasta junio de 2015. Fueron observadas 649 admisiones en el periodo y 68 pacientes con lesiones, representando 10,47\% de incidencia. Entre esos pacientes, 51,5\% fueron hombres y 48,5\% mujeres. 58,8\% de las lesiones ocurrieron en los primeros 14 días de admisión. Los pacientes con más de 60 años fueron la mayoría (79,4\%). Entre los individuos con más de 60 años, 61,1\% presentaron alto riesgo para el desarrollo de las lesiones por presión. La región sacra fue el sitio más común (46,4\%). La fase 2 de las lesiones fue la más común (61,9\%). Esto estudio mostró la baja incidencia de lesiones de presión en los enfermos de la Unidad de Cuidados Intensivos, en comparación con los estudios nacionales e internacionales. El estudio también indicó que las lesiones se producen más en sitios anatómicos considerados de mayor presión, y entonces luego encontradas en etapas tempranas.

DESCRIPTORES: Heridas y traumatismos. Úlcera por Presión. Unidad de Cuidados Intensivos. Incidencia. Estomaterapia.

\section{INTRODUÇÃO}

As Unidades de Terapia Intensiva (UTI) são ambientes destinados ao atendimento de pacientes graves, com potencial risco de morte, que necessitam de atendimento ininterrupto. São caracterizadas, muitas vezes, como um ambiente relacionado ao sofrimento e à morte ${ }^{1}$. Ali se desenvolve tratamento intensivo e hostil, pois, além da situação crítica em que o paciente se encontra, existem fatores prejudiciais à sua estrutura psicológica, como falta de condições favoráveis ao sono, intervenções terapêuticas frequentes, isolamento, permanência no leito por um período longo e medo do agravamento da doença e da própria morte. Alguns desses fatores favorecem o surgimento de complicações como atrofias musculares e úlceras por pressão ${ }^{2}$.

Recentemente o National Pressure Ulcer Advisory Panel (NPUAP) padronizou um novo conceito sobre úlcera por pressão (lesão por pressão) e seu estadiamento. Atualmente, a úlcera por pressão é denominada "lesão por pressão" e é definida como: "dano localizado na pele e/ou tecidos moles subjacentes, geralmente sobre uma proeminência óssea ou relacionada ao uso de dispositivo médico ou a outro artefato. A lesão pode se apresentar em pele íntegra ou como úlcera aberta, e pode ser dolorosa. A lesão ocorre como resultado da pressão intensa e/ou prolongada em combinação com o cisalhamento. A tolerância do tecido mole à pressão e ao cisalhamento pode também ser afetada pelo microclima, nutrição, perfusão, comorbidades e pela sua condição"3.

Apesar da modernização dos cuidados de saúde, a incidência e prevalência de lesões por pressão em UTI permanecem elevadas. Estudo realizado em 22 UTIs, de 15 hospitais de Belo Horizonte, identificou que a ocorrência de pelo menos uma lesão por pressão por paciente foi de $35,2 \%{ }^{4}$. Na literatura internacional, há uma prevalência que varia de 4,0 a 49,0\% e uma incidência de 3,8 a 12,4\% em ambiente de cuidados intensivos ${ }^{5}$.

As lesões por pressão são feridas que podem ser causadas por fatores intrínsecos e extrínsecos ao paciente. Como fatores extrínsecos, têm-se a pressão, o cisalhamento e a umidade. A pressão é considerada o principal fator causador, pois o seu efeito patológico no tecido pode ser atribuído à intensidade da pressão, à sua duração e tolerância tecidual ${ }^{6}$. Dentre os fatores intrínsecos, destacam-se: a idade, o estado nutricional, a perfusão tecidual, o uso de alguns medicamentos e as doenças crônicas (como diabetes melito e doenças cardiovasculares) ${ }^{7}$. 
Essas lesões são consideradas eventos adversos ocorridos durante a hospitalização e refletem de forma indireta a qualidade do cuidado prestado. Além disso, são uma complicação frequente em pacientes graves e têm grande impacto sobre sua recuperação e qualidade de vida ${ }^{8}$. Portanto, cabe aos profissionais de saúde, em especial à equipe de Enfermagem, identificar os fatores de risco para o seu desenvolvimento nos pacientes e planejar ações de caráter preventivo, a fim de melhorar a qualidade da assistência que lhes é devida.

Para avaliação do risco de formação da lesão por pressão, existem diversas escalas; vale destacar a Escala de Braden, que foi desenvolvida com base na fisiopatologia da lesão por pressão. De acordo com tal escala, são avaliados seis fatores de risco (subescalas) no paciente, que são:

1. Percepção sensorial: referente à capacidade de o paciente reagir significativamente ao desconforto relacionado à pressão;

2. Umidade: refere-se ao nível em que a pele é exposta à umidade;

3. Atividade: avalia o grau de atividade física;

4. Mobilidade: refere-se à capacidade do paciente em mudar e controlar a posição do seu corpo;

5. Nutrição: retrata o padrão usual de consumo alimentar do paciente;

6. Fricção e cisalhamento: retrata a dependência do paciente para a mobilização e posicionamento e sobre estados de espasticidade, contratura e agitação que podem levar à constante fricção.

Cada subescala tem pontuação que varia entre um e quatro, com exceção do domínio "fricção e cisalhamento". A somatória total fica entre os valores 6 e 23, sendo que quanto menor o escore, maior o risco de ocorrência de lesões ${ }^{9}$.

As instituições hospitalares, preocupadas com a segurança e a qualidade de sua assistência, utilizam instrumentos, como a escala de Braden, com o intuito de auxiliar os enfermeiros no processo de decisão das medidas preventivas a serem adotadas, de acordo com o risco de cada paciente ${ }^{10}$.

Outra forma de buscar qualidade e segurança na assistência é o processo voluntário de acreditação hospitalar. A instituição prestadora de serviço é submetida a uma avaliação criteriosa por uma organização não governamental, sem fins lucrativos e de interesse coletivo, chamada de Organização Nacional de Acreditação (ONA). O principal objetivo dela é a implantação nacional de um processo permanente de melhoria da qualidade da assistência à saúde, estimulando todos os serviços de saúde a atingirem padrões mais elevados de qualidade, dentro do processo de acreditação ${ }^{11}$.

As instituições hospitalares passam por um processo de avaliação e visita dos avaliadores, no qual é gerado um parecer final sobre a indicação para acreditação. O certificado é emitido pela Instituição Acreditadora, estabelecendo um nível de classificação: "Acreditado", "Acreditado pleno" e "Acreditado com Excelência". Os dois primeiros têm validade de dois anos e o último, de três $\operatorname{anos}^{12}$. Ao final desse período, a instituição deve submeter-se a um novo processo de avaliação.

Diante da adesão à acreditação, a assistência de Enfermagem é essencial para um programa de qualidade, devido à numerosa quantidade de profissionais e por sua atuação direta e permanente aos pacientes. A Enfermagem atua com autonomia e corresponsabilidade, por meio de instrumentos da estrutura organizacional (como regimento interno, protocolos assistenciais, organograma, rotinas, sistemas de comunicação e controle) $)^{13,14}$.

Tais protocolos proporcionam maior efetividade e eficiência na prestação de serviços em saúde. Assim, os processos de acreditação são influenciados pelas ações da Enfermagem e, ao mesmo tempo, têm importantes implicações no cotidiano de trabalho da equipe.

A preocupação acerca da temática é de ampla magnitude e envolve tanto recursos financeiros quanto humanos em seus aspectos de prevenção, tratamento e aprimoramento de tecnologias de cuidado de Enfermagem. Assim, constitui um grande desafio a ser enfrentado pela equipe multiprofissional envolvida na assistência prestada nas UTIs ${ }^{15}$.

A importância do problema está no fato de que a prevenção da lesão por pressão tem sido considerada um indicador de qualidade não só do serviço de saúde como também da assistência de Enfermagem na UTI. Devido à gravidade do problema que essas lesões podem ocasionar, as mesmas foram incluídas pela Agency for Health Care Polycy and Research (ACPHR) como um dos indicadores de qualidade da assistência à saúde ${ }^{15}$.

Diante da complexidade do problema e considerando a relevância do assunto em questão na realidade da UTI (visto que estes pacientes desenvolvem lesão por pressão sob os cuidados da instituição - seja por uma estrutura deficiente, instabilidade hemodinâmica, ou por uma deficiência no cuidar), realizou-se este estudo. Desse modo, o trabalho contribuirá para a produção de conhecimento à comunidade científica, principalmente aos profissionais de saúde 
que cuidam de pacientes gravemente enfermos. Portanto, o objetivo deste estudo foi analisar o perfil de incidências das lesões por pressão em UTI Adulto em 2014.

\section{MÉTODOS}

Trata-se de uma pesquisa de abordagem quantitativa, usando um eixo descritivo, retrospectivo e documental, o qual consistiu na análise documental das fichas do gerenciamento de riscos de lesões por pressão nos pacientes da UTI Adulto.

A pesquisa foi realizada em um hospital de atenção secundária dentro da rede pública de saúde. Esse foi o primeiro hospital público das regiões Norte e Nordeste a receber o título de acreditação hospitalar nível 1 e 2 pela ONA. Trata-se de um hospital de apoio para a rede terciária de assistência do estado do Ceará, atendendo exclusivamente a clientela do Sistema Único de Saúde (SUS). Oferece à população 336 leitos, distribuídos nas clínicas médicas, cirúrgica, pediátrica, Unidade de Cuidados Especiais, UTI adulto (21 leitos), UTI neonatal, UTI pediátrica e berçário médio risco. Desenvolve, ainda, programas de assistência ambulatorial e domiciliar, como também cuidados especiais e personalizados, com equipes multidisciplinares para pacientes portadores de enfermidades crônicas.

Os dados foram coletados por meio de um roteiro junto às fichas de monitoramento de riscos, alimentadas diariamente por acadêmicos de Enfermagem do serviço de estomaterapia e supervisionadas pela estomaterapeuta da instituição. Enfatiza-se que a coleta de dados foi realizada após treinamento dos acadêmicos de Enfermagem acerca do monitoramento de lesões por pressão e dados de incidência. Utilizou-se o cálculo universal para a análise de percentual das incidências, sendo este: número de casos novos de lesões por pressão na UTI sobre o número de pessoas expostas ao risco na unidade, no mesmo período, multiplicado por 100.

A ficha de monitoramento de pele contém informações quanto aos riscos clínicos que cada paciente apresenta, dentre eles o risco para lesão por pressão segundo a escala de Braden, além do tempo de internação do paciente no hospital e na UTI, e se há integridade da pele ou não no momento da admissão na unidade.

Os dados obtidos foram acerca das incidências de lesões por pressão na UTI referentes ao período de janeiro a dezembro de 2014. A coleta e organização dos dados foram realizadas de maio a junho de 2015, tendo sido utilizado como instrumento de coleta de dados um formulário elaborado pelos pesquisadores, no qual se questionava: a incidência da lesão por pressão; o mês de ocorrência; o sexo do paciente; a idade; o tempo de internação na unidade; a localização da lesão; o estágio e o risco na escala de Braden.

Para a análise, os dados foram transcritos e tabulados em uma planilha do programa Excel do Windows 7 Profissional e, posteriormente, organizados em tabelas e figuras. Eles foram interpretados e fundamentados com base na literatura pertinente à temática.

A incidência expressa o número de casos novos, na população, durante um determinado período. Para estabelecê-la, é necessário especificar a duração do tempo de observação de surgimento dos casos novos. Dessa forma, a incidência reflete a dinâmica com que os casos aparecem no grupo e a força da morbidade (mortalidade). Para o cálculo da incidência, foi utilizada a Equação $1^{16}$ :

Índice de incidência $=\mathrm{n}^{\circ}$ de "casos novos" em determinado período x $100 / \mathrm{n}^{\circ}$ de pessoas expostas ao risco no mesmo período.

Foram respeitadas as normas éticas e legais da Resolução do Conselho Nacional de Saúde (CNS) N 466/12, que regulamenta pesquisa envolvendo seres humanos, incorporando, sob a ótica do indivíduo e das coletividades, os referenciais básicos da Bioética, autonomia, não maleficência, beneficência e justiça. Visa, sobretudo, a assegurar os direitos e deveres da comunidade científica, dos sujeitos da pesquisa e do estudo. Foi resguardado o anonimato dos integrantes da pesquisa, que foi aprovada pelo Comitê de Ética em Pesquisa em Seres Humanos da Universidade de Fortaleza, sob o parecer $n^{\circ} 1.188 .404$.

\section{RESULTADOS}

Em 2014, houve um total de 649 internações na UTI adulto. Todos os pacientes admitidos nela apresentaram algum risco de desenvolvimento de lesão por pressão, segundo a escala de Braden. A partir das fichas de gerenciamento de riscos clínicos, foram identificadas 68 notificações de incidências na referida UTI, as quais compuseram a amostra da pesquisa.

As 68 notificações corresponderam a uma incidência de 10,47\% do total de internações nesta unidade em 2014. Foi observado que, entre os casos notificados de lesão por 
pressão, 35 dos pacientes $(51,5 \%)$ eram do sexo masculino e $33(48,5 \%)$ do feminino, conforme Tabela 1.

Acerca da distribuição dos pacientes quanto ao tempo de internação até o surgimento da lesão por pressão, observou-se que, dos 68 pacientes que desenvolveram a lesão por pressão durante a internação na UTI, $40(58,8 \%)$ incidiram durante os primeiros 14 dias de internação no leito, $18(26,5 \%)$ apresentaram lesão entre o $15^{\circ}$ e o $30^{\circ}$ dia, $6(8,9 \%)$ entre o $31^{\circ}$ e o $45^{\circ}$ dia, e apenas $4(5,8 \%)$ apresentaram incidência após o $45^{\circ}$ dia de internação, os dados estão descritos na Tabela 2.

Tabela 1. Distribuição dos pacientes do estudo de acordo com o sexo.

\begin{tabular}{lcc}
\hline Sexo dos pacientes & $\mathbf{n}$ & $\%$ \\
\hline Feminino & 33 & 48,5 \\
\hline Masculino & 35 & 51,5 \\
\hline
\end{tabular}

Tabela 2. Distribuição dos pacientes quanto ao tempo de internação até o surgimento da lesão por pressão

\begin{tabular}{lcc}
\hline Dias de internação & $\mathbf{n}$ & $\%$ \\
\hline 0 a 14 dias & 40 & 58,8 \\
\hline 15 a 30 dias & 18 & 26,5 \\
\hline 31 a 45 dias & 6 & 8,9 \\
\hline+45 dias & 4 & 5,8 \\
\hline
\end{tabular}

$\mathrm{Na}$ Tabela 3, relacionam-se os dados da faixa etária e os valores encontrados na avaliação do risco dos pacientes utilizando-se a escala de Braden. Assim, foram obtidos os seguintes resultados entre os 68 pacientes que apresentaram a lesão durante a internação na unidade: a frequência predominante quanto à faixa etária foi acima de 60 anos em 54 pacientes (79,4\%); seguido de 10 na faixa etária de 41 a 60 anos (14,7\%); e 4 de 20 a 40 anos (5,9\%). Dos indivíduos com faixa etária acima de 60 anos, $33(61,1 \%)$ apresentaram risco elevado para o desenvolvimento de lesão por pressão, e 16 (29,6\%), risco muito elevado.

Quanto à classificação em estágios, conforme Tabela 4: $52(61,9 \%)$ das lesões por pressão notificadas eram de estágio 2; 17 (20,2\%) de estágio 1; e 12 (14,2\%) de estágio indeterminado. Pode-se observar a ocorrência das lesões quanto à região anatômica do seu desenvolvimento. Percebe-se maior incidência das lesões na região sacral, vistas em 39 (46,4\%) dos casos, seguidas de calcâneo, em 25 (29,76\%) e, em terceiro lugar, trocânter, em 8 (9,5\%) dos casos. Quanto ao estadiamento destas lesões, houve predomínio do estágio 2 nos 3 locais, conforme citado.

\section{DISCUSSÃO}

As lesões por pressão representam uma das principais complicações que acometem pacientes críticos hospitalizados. São considerados pacientes críticos aqueles que têm condições

Tabela 3. Classificação do risco pela escala de Braden dos pacientes na unidade do campo de estudo, de acordo com a faixa etária.

\begin{tabular}{|c|c|c|c|c|c|}
\hline \multirow{2}{*}{$\begin{array}{l}\text { Faixa etária } \\
\text { (anos) }\end{array}$} & \multicolumn{2}{|c|}{ Pacientes } & \multirow{2}{*}{$\begin{array}{l}\text { Classificação do risco } \\
\text { segundo a escala de Braden }\end{array}$} & \multicolumn{2}{|c|}{ Pacientes } \\
\hline & $\mathrm{n}$ & $\%$ & & $\mathrm{n}$ & $\%$ \\
\hline & & & Moderado & 0 & 0 \\
\hline \multirow[t]{3}{*}{20 a 40} & 4 & 5,9 & Elevado & 4 & 5,9 \\
\hline & & & Muito elevado & 0 & 0 \\
\hline & & & Moderado & 0 & 0 \\
\hline \multirow[t]{3}{*}{41 a 60} & 10 & 14,7 & Elevado & 4 & 5,9 \\
\hline & & & Muito elevado & 6 & 8,8 \\
\hline & & & Moderado & 5 & 7,4 \\
\hline \multirow[t]{2}{*}{ Acima de 60} & 54 & 79,4 & Elevado & 33 & 61,1 \\
\hline & & & Muito elevado & 16 & 29,6 \\
\hline
\end{tabular}

Fonte: Dados da pesquisa. 
Tabela 4. Distribuição da localização da incidência das lesões por pressão na unidade do campo de estudo, considerando a localização anatômica e o estágio de classificação.

\begin{tabular}{lcccccccc}
\hline \multirow{2}{*}{$\begin{array}{l}\text { Localização } \\
\text { anatômica }\end{array}$} & \multicolumn{4}{c}{ Estadiamento da lesão por pressão } & \multicolumn{2}{c}{ Total } \\
\cline { 2 - 7 } & $\mathbf{1}$ & $\mathbf{2}$ & $\mathbf{3}$ & $\mathbf{4}$ & Indeterminada & LPTP & $\mathrm{n}$ & $\%$ \\
\hline Sacral & 6 & 29 & 1 & 0 & 3 & 0 & 39 & 46,4 \\
\hline Occipital & 1 & 0 & 0 & 0 & 1 & 0 & 2 & 2,4 \\
\hline Calcâneo & 7 & 10 & 0 & 0 & 7 & 1 & 25 & 29,76 \\
\hline Panturrilha & 0 & 3 & 1 & 0 & 1 & 0 & 5 & 6,0 \\
\hline Trocânter & 2 & 6 & 0 & 0 & 0 & 0 & 8 & 9,5 \\
\hline Joelho & 1 & 1 & 0 & 0 & 0 & 0 & 2 & 2,4 \\
\hline Coluna vertebral & 0 & 1 & 0 & 0 & 0 & 0 & 1 & 1,1 \\
\hline Pavilhão auricular & 0 & 2 & 0 & 0 & 0 & 0 & 2 & 2,4 \\
\hline Total & 17 & 52 & 2 & 0 & 12 & 1 & 84 & 100 \\
\hline
\end{tabular}

LPTP: lesão por pressão tissular profunda.

Fonte: Dados da pesquisa.

clínicas graves ou necessidade de controles mais frequentes e rigorosos, associados a terapias de maior complexidade, de caráter invasivo ou não ${ }^{17}$.

Essas feridas prolongam a hospitalização, dificultam a recuperação do doente e aumentam o risco para o desenvolvimento de outras complicações, tais como infecções ou osteomielite. Mas é por representarem um acréscimo ao sofrimento físico e emocional desses pacientes que as lesões por pressão merecem atenção por parte da equipe multiprofissional, no sentido de prevenir o seu aparecimento ou favorecer o seu tratamento ${ }^{17}$.

Segundo dados da NPUAP ${ }^{3}$, a prevalência de lesões por pressão em hospitais é de 15,0\% dos pacientes admitidos, e a incidência é de 7,0\%. No Reino Unido, casos novos de lesões por pressão acometem entre 4,0 a $10,0 \%$ das admissões. No Brasil, embora existam poucos trabalhos sobre incidência e prevalência, estudo realizado em hospital geral universitário evidenciou uma incidência de 39,81\%. Já em relação à incidência em UTI, estudos nacionais apontaram valores que variam de 23,1 a $62,5 \%{ }^{18-20}$. Face ao exposto, o resultado de $10,47 \%$ identificado para incidência de lesão por pressão na UTI investigada encontra-se abaixo do esperado para as unidades hospitalares e centros de terapia intensiva.

Essa baixa incidência encontrada pode ser atribuída ao conjunto de fatores percebidos na instituição do estudo, como a presença de programas de prevenção direcionados para profissionais das UTIs, orientados pelo serviço de estomaterapia e monitorados pelo gerenciamento de riscos clínicos; nesses programas, contamos com a atuação de acadêmicos de Enfermagem em visitas sistemáticas nas unidades.

A instituição em estudo aderiu ao programa de qualidade contínuo norteado pela ONA e aprimorou as diretrizes recomendadas pelo Protocolo de Prevenção de Úlcera do Plano Nacional de Segurança do Paciente. Nesse cenário, a lesão por pressão se destaca como indicador importante e que deve ser mensurado e trabalhado nas instituições, principalmente nas unidades de maior risco, como é o caso das UTIs.

O hospital possui serviço de estomaterapia em funcionamento desde 2004, responsável tanto pela construção/ atualização de protocolos de assistência para prevenção de pele, quanto pela avaliação e identificação dos pacientes em risco. Desse modo,é possível nortear a escolha das intervenções preventivas e curativas adequadas, a exemplo da utilização a escala de Braden como instrumento de avaliação de risco realizada pelo enfermeiro diariamente. O serviço conta ainda com atividades de capacitação dos profissionais nos programas de educação continuada com foco na identificação, descrição e documentação dos registros adequados referentes à lesão por pressão e ao fornecimento de materiais e equipamentos necessários para a prevenção. Logo, denota-se uma reflexão acerca da qualidade da assistência prestada.

O protocolo de prevenção de lesões de pele da instituição em estudo define como principais estratégias: mudança 
de decúbito a cada três horas; colchão pneumático para $100 \%$ dos pacientes da UTI; acompanhamento semanal pela estomaterapeuta; utilização de ficha de monitoramento de pele com registro da condição da pele na admissão e registro da avaliação diária da pele; envolvimento e sensibilização da equipe multiprofissional; e redução de fricção e cisalhamento por meio da oferta e do uso de dispositivos, como filme transparente em proeminências ósseas e hidratação diária da pele após o banho. Tais medidas se fizeram eficientes na redução da incidência.

Achados semelhantes, quanto à baixa incidência de lesão por pressão, foram identificados em estudo ${ }^{21}$ realizado na UTI de hospital universitário que encontrou uma incidência de 10,6\%, que é muito próxima da encontrada no presente estudo, e considerada elevada em comparação com as detectadas nos estudos realizados em instituições privadas, as quais normalmente apresentam índices mais baixos.

No presente estudo, foi identificado que o período das duas primeiras semanas de internação é o tempo de maior frequência para o surgimento de lesões relacionadas à pressão. A tendência de desenvolvimento de lesão por pressão nas duas primeiras semanas de hospitalização é também encontrada em outros estudos ${ }^{22-25}$. Considera-se, portanto, esse período como um fator de risco para o desenvolvimento desse tipo de lesão. Faz-se necessário e essencial a atuação do enfermeiro na linha de cuidados do paciente crítico na adoção precoce das medidas preventivas. Destaca-se a importância de ter processos de trabalho definidos para a detecção do risco para lesão nas primeiras seis horas de internamento para permitir que as medidas sejam adotadas o mais brevemente possível. É imprescindível a realização da avaliação do risco na admissão do paciente.

Pode-se inferir que a população estudada foi predominantemente idosa, ou seja, apresentando uma maior suscetibilidade ao surgimento de lesão por pressão devido às características ocasionadas pelo envelhecimento da pele.

Numerosas são as mudanças que se observa na pele com o avanço da idade. Temos como sinais clínicos clássicos do envelhecimento: afinamento, fragilidade, rugas, queratose e desidratação. Tais fatores ocorrem devido à diminuição da camada dérmica, da sua vascularização, da proliferação epidérmica e de suas funções como a percepção da dor, a resposta inflamatória e a função de barreira, tornando-a mais vulnerável à injúria ${ }^{26}$.
Estudos indicam que o progressivo comprometimento da atividade celular na terceira idade, especialmente dos fibroblastos, afeta diretamente a produção de colágeno local, principal proteína responsável pela formação da estrutura e força tensil tegumentar. Assim, verifica-se um aumento da probabilidade de doenças crônicas, muitas das quais tornam as pessoas mais suscetíveis a desenvolver lesões por pressão $0^{26-28}$.

De acordo com os resultados para classificação de risco segundo a escala de Braden, houve predomínio de pacientes que desenvolveram a lesão com risco elevado para lesões por pressão na UTI.Diante disto, a utilização dessa escala permite identificar fatores de risco específicos de cada paciente, o que pode contribuir para a percepção das limitações presentes em diferentes grupos de pacientes, independentemente de qual seja o diagnóstico ou tipo de patologia ${ }^{7}$.

As diretrizes internacionais e nacionais aconselham a utilização da escala de Braden para contribuir na identificação dos pacientes que apresentam o risco para lesão por pressão desde admissão e durante o período de internação e aplicação das medidas preventivas pelos profissionais. Sugere-se que a reavaliação ocorra pelo menos a cada 48 horas após admissão ou sempre que as condições do paciente se modificarem ${ }^{29}$.

Quanto à localização anatômica das úlceras, constatou-se que as áreas mais frequentes foram: sacral, calcâneo e trocânter. Estas correspondem aos pontos de maior pressão em paciente na posição dorsal. Sabendo que existe relação direta entre a localização mais frequente de lesão por pressão e a posição em que o paciente permanece por um maior período, pode-se concluir que os pacientes mantiveram-se no leito por um tempo prolongado na posição decúbito dorsal, favorecendo o desenvolvimento da lesão. Tais fatos nos remetem à necessidade de avaliação e registro por parte da equipe acerca da condição hemodinâmica do paciente, bem como à importância de programação da mudança de decúbito conforme condição clínica, que deve ser avaliada a cada troca de plantão.

Houve predomínio de lesão por pressão em estágio 2, o que aponta que estes resultados coincidem com estudos ${ }^{30}$ realizados em hospitais americanos, nos quais $90,0 \%$ das lesões eram de estágios 1 e 2; e com estudos ${ }^{21,31}$ nacionais, desenvolvidos em hospitais universitários, que também mostraram predomínio desses mesmos estágios. Tal característica é reflexo da atenção dos enfermeiros quanto à questão da identificação e notificação precoce de lesões por pressão durante o período de internação. 


\section{CONCLUSÃO}

O estudo evidencia uma baixa incidência de lesão por pressão nos pacientes internados na UTI, levando-se em consideração o referencial de estudos internacionais e nacionais. Observa-se, também, que predomina o surgimento de lesões em localizações anatômicas consideradas de maior pressão e que estas são identificadas precocemente.

Embora exista a atuação eficaz da equipe de Enfermagem na aplicação da escala de Braden na identificação em tempo hábil dos pacientes que apresentam risco de desenvolvimento de lesões por pressão, ainda se faz necessário estabelecer estratégias para a prevenção das mesmas e sempre avaliar a adesão ao protocolo estabelecido. Tais medidas visam a minimizar as complicações para o paciente e, consequentemente, reduzir a incidência na unidade, reforçando as ações do protocolo de prevenção de lesões de pele que necessitam ser intensificadas.

Percebe-se a importância de identificar o nível de adesão à prevenção da lesão por pressão dos profissionais de um modo geral, e não somente da Enfermagem. Nesse processo, é necessário tentar identificar as falhas não só nas atribuições da equipe de Enfermagem, mas também nos papéis que cabem ao médico, ao nutricionista, ao fisioterapeuta e a outros profissionais envolvidos na logística da prevenção (como a eficiência da farmácia e engenharia clínica). Assim, é essencial identificar a interação de protocolos e fazer ajustes nas ações previstas que têm impacto ou ação direta no comprometimento da pele do paciente criticamente enfermo.

\section{REFERÊNCIAS}

1. Proença MO, Dell Agnolo CM. Internação em Unidade de Terapia Intensiva: percepção de pacientes. Rev Gaúcha Enferm. 2011;32(2):279-86.

2. Nogueira PC, Caliri MH, Haas VJ. Profile of patients with spinal cord injuries and occurrence of pressure ulcer at a university hospital. Rev Latino-Am Enferm. 2006;4(3):372-7.

3. National Pressure Ulcer Advisory Panel (NPUAP) [Internet] [cited 2016 Jun 22]. Available from: http://www.npuap. org/resources/educational-and-clinical-resources/npuappressure-injury-stages/

4. Gomes FS, Bastos MA, Matozinhos FP, Temponi HR, Velásquez-Meléndez G. Fatores associados à úlcera por pressão em pacientes internados nos Centros de Terapia Intensiva de Adultos. Rev Esc Enferm USP. 2010;44(4):1070-6.

5. Shahin ES, Dassen T, Halfens RJ. Prevalência de pressão úlcera e incidência em pacientes de terapia intensiva: revisão da literatura. Nurs Crit Care. 2008;13(2):71-9.

6. Bryant RA. Acute \& chronic wounds: nursing management. 2. ed. Michigan: Mosby; 2000. 558p.

7. Bergstrom N, Braden BJ, Kemp M, Champagne M, Ruby E. Multi-site of incidence of pressure ulcers and the relationship between risk level, demographic characteristics, diagnoses, and prescription of preventive interventions. J Am Geriatr Soc. 1996;44:22-30.

8. Silva EW, Araújo RA, Oliveira EC, Falcão VT. Aplicabilidade do protocolo de prevenção de úlcera de pressão em unidade de terapia intensiva. Rev Bras Ter Intensiva. 2010;2(22):175-85.

9. Ayello EA, Braden B. How and why to do pressure ulcer risk assessment. Adv Skin Wound Care. 2002;15(3):125-33.

10. Fernandes LM, Caliri MHL. Uso da escala de Braden e Glasgow para identificação do risco para úlcera de pressão em pacientes internados e centro de terapia intensiva. Rev Latino-Am Enferm. 2008;6(16):1-6.
11. Organização Nacional de Acreditação. A saúde no Brasil: agora tem um processo permanente de avaliação e certificação da qualidade. [Folder]. Brasília (DF); 2000.

12. Quinto NA, Bittar OJ. Hospitais: administração da qualidade e acreditação de organizações complexas. Porto Alegre: Dacasa; 2004

13. Lima SB, Erdmann AL. A enfermagem no processo da acreditação hospitalar em um serviço de urgência e emergência. Acta Paul Enferm. 2006;19(3):271-8.

14. Vargas MA, Albuquerque GL, Erdman AL, Ramos FR. Onde (e como) encontramos a qualidade no serviço de enfermagem hospitalar? Rev Bras Enferm. 2007;60(3):339-43.

15. Souza TS, Maciel OB, Méier MJ, Danski MTR, Lacerda MR. Estudos clínicos sobre úlcera por pressão. Rev Bras Enferm. 2010;3(63):470-6.

16. Pereira MG. Morbidade. In: Pereira MG. Epidemiologia: teoria e prática. Rio de Janeiro: Guanabara Koogan; 2005. p. 76-101.

17. Blanes L, Duarte IS, Calii JA, Ferreira LM. Avaliação clínica e epidemiológica das úlceras por pressão em pacientes internados no hospital São Paulo. AMB Rev Assoc Med Bras. 2004;50(2):182-7

18. Rogenski NM, Kurcgant P. Incidência de úlceras por pressão após a implementação de um protocolo de prevenção. Rev Latino-Am Enferm. 2012;20(2):[07 telas]. Available from: http://www.scielo.br/pdf/rlae/v20n2/pt_16.

19. Louro M, Ferreira M, Povoa P. Avaliação de protocolo de prevenção e tratamento de úlceras por pressão. Rev Bras Ter Intensiva. 2007;19(3):337-41.

20. Fernandes NC, Torres GV. Incidência e fatores de risco de úlceras por pressão em pacientes de unidade de terapia intensiva. Cienc Cuid Saúde. 2008;7(3):304-10. Available from: http://eduem.uem.br/ojs/index.php/CiencCuidSaude/ article/view/6484. 
21. Rogenski NM, Santos VL. Estudo sobre a incidência de úlceras por pressão em um hospital universitário. Rev Latino-Am Enferm. 2005;13(4):474-80.

22. Cardoso MC, Caliri MH, Hass VJ. Prevalência de úlcera de pressão em pacientes críticos internados em um hospital universitário. Rev Min Enferm. 2004;8(2):316-20.

23. Blanes L, Duarte IS, Calil JA, Ferreira LM. Avaliação clínica e epidemiológica das úlceras por pressão em pacientes internados no hospital São Paulo. AMB Rev Assoc Med Bras. 2004;50(2):182-7.

24. Paranhos WY, Santos VL. Avaliação de risco para úlceras de pressão por meio da Escala de Braden, na língua portuguesa. Rev Esc Enferm USP. 1999;33(esp.):191-206.

25. Costa IG, Caliri MH. Incidência de úlcera de pressão em centro de terapia intensiva de um hospital universitário e fatores de risco relacionados. Rev Paul Enferm. 2004;23(3/4):202-7.

26. Yamada BF. Pele - o manto protetor: higiene e hidratação. São Paulo: Andreoli; 2015. p. 57-64.
27. Santos VL. Avanços tecnológicos no tratamento de feridas e algumas aplicações em domicílio. In: Duarte YA, Diogo MJ. Atendimento domiciliar: um enfoque gerontológico. São Paulo: Atheneu; 2000. p. 265-306.

28. Mulder GD. Factors complicating wound repair. In: Kloth LC, McCullock JM, Freedar JA. Wound healing alternatives in management. Philadelphia: F.A. Davis Company; 1990. p. 43-52.

29. Wound Ostomy and Continence Nursing Society. Guideline for Prevention and Management of Pressure ulcers. WOCN Clinical Practice Guideline Series. Glenview; 2003. 52p.

30. Whittington $K$, Patrick M, Roberts JL. A national study of pressure ulcer prevalence and incidence in acute care hospitals, J Wound Care.. 2000;27(4):209-15.

31. Costa IG, Caliri MH. Incidência de úlceras de pressão em centro de terapia intensiva de um hospital universitário e fatores de risco relacionados. Rev Paul Enferm. 2005;23(3/4):202-7. 